\title{
ABORDAGENS METODOLOGICAS NO CAMPO DA PESQUISA CIENTIFICA
}

\author{
Keila Aparecida Marques ${ }^{1}$; Ana Flávia Ferreira de Melo² $^{2}$
}

\section{Resumo}

A crescente demanda por novos conhecimentos acarreta em inúmeros movimentos no campo das pesquisas cientificas, no que tange a escolha da metodologia a ser utilizada no entendimento dos fenômenos estudados. Com o objetivo em apresentar uma compreensão sobre a abordagem metodológica quantitativa nas pesquisas cientificas, foram encontrados resultados que fortalecem 0 entrelaçamento das duas abordagens metodológicas que norteiam o universo da pesquisa cientifica. No entanto nenhum método pode ser considerado melhor em detrimento do outro, pois são consideradas as vantagens e desvantagens na utilização entre os métodos e também as características latentes que diferem os métodos e suas particularidades. Estas considerações consistem na relevância destacada neste estudo apresentado, em que abordagens quantitativas e qualitativas são percebidas entre os pesquisadores como complementares no universo da pesquisa cientifica.

Palavras Chave: Métodos Quantitativos, Complementaridade, Pesquisa Cientifica.

\footnotetext{
${ }^{1}$ Administradora e Mestranda em Gestão Organizacional pela Universidade Federal de Goiás.Catalão - Goiás/Brasil. e-mail: keilamarques595@gmail.com ${ }^{2}$ Contadora e Mestranda em Gestão Organizacional pela Universidade Federal de Goiás. Catalão - Goiás/Brasil. e-mail: anflaviafms@hotmail.com
} 


\section{Introdução}

O saber científico na visão de Minayo e Sanches (2003) consiste na busca por estratégias entre a teoria e a realidade empírica, o método científico que conduz o pesquisador ao fenômeno tem sido visto como sendo o 'fio condutor' para essa articulação, a partir desta premissa constata-se a relevância dos métodos quantitativos e qualitativos.

Ainda na compreensão destes autores, o intuito não está voltado em medir o grau de importância entre os dois métodos, o qualitativo e o quantitativo, uma vez que nenhuma das duas abordagens são capazes de suprir com eficácia a compreensão completa da realidade. E para ser considerado como um bom método, deve se avaliar qual é o método mais adequado ao objeto de investigação e de ofertar princípios teóricos para a análise, de forma que o método escolhido pelo pesquisador tenha condições de executar e operacionalizar o estudo proposto.

Neste estudo, o foco está voltado aos conhecimentos sobre os métodos quantitativos, no entanto a complementaridade entre estas duas abordagens ocupa lugar de destaque na busca por conhecimento cientifico, Minayo e Sanches (2003) destaca a grande importância na escolha de um destes métodos ou da utilização destes dois construtos qualitativos e quantitativos simultaneamente.

O método quantitativo surgiu das ciências naturais e do positivismo, sendo este o estabelecimento de cientificidade, ou seja, de quais formas o pesquisador pode estudar os fenômenos, que tinham como fundamento 0 conhecimento da natureza através do método científico, o qual era capaz de controlar e manipular as variáveis que modificam o objeto de estudo, Silva (2010).

A delimitação da abordagem quantitativa em pesquisa, impõem algumas situações distintas que devem estar presentes desde a elaboração do problema, o que implica uma relevância da revisão da literatura, da coleta e a análise dos dados e do desenvolvimento do relatório de resultados (Sampieri; Collado; Lucio, 2013).

Conforme Gabriel (2014), cuidados especiais podem e devem ser adotados em todo o desenvolvimento do relatório de pesquisa para diminuir as falhas ocasionadas através utilização incorreta de determinada análise estatística ou de técnica multivariada.

Fakis et al. (2014) em uma publicação recente determina o uso de estatística para mensurar dados qualitativos encontrados em questionários a partir de um documento específicos. Portanto, concluem sobre a importância de elaborar um novo contexto que propõe a utilização de um método estatístico eficaz para explorar as informações obtidas.

Em contrapartida Bignardi (2009) tem seu entendimento na pesquisa quantitativa, a qual é concentrada na dimensão mensurável da realidade, tendo seu início na visão newtoniana dos fenômenos e circula com eficiência no âmbito dos extratos mais densos e materiais da realidade. Seus resultados facilitam 0 planejamento de atitudes coletivas sendo passível de generalizações, sobretudo quando as populações evidenciadas representam com fidelidade o coletivo.

A pesquisa quantitativa tem seu foco voltado na objetividade, acredita que a realidade só pode ser compreendida com base na análise de dados 
brutos, coletados com o apoio de instrumentos padronizados e neutros. Como as amostras comumente são grandes e consideradas representativas da população, os resultados demonstram um retrato real de toda população alvo da pesquisa, Fonseca (2012) ainda continua corroborando que a pesquisa quantitativa tem suas bases na linguagem matemática, no intuito de descrever as causas de um fenômeno, relações entre variáveis, entre outros.

Embasado nos discursos dos autores citados anteriormente, está pesquisa se propõe apresentar uma compreensão sobre a abordagem metodológica quantitativa nas pesquisas cientificas, sabe-se que o estudo quantitativo tem suas raízes no pensamento positivista lógico, com ênfase no raciocínio dedutivo, nas regras da lógica e atributos mensuráveis da experiência humana.

Assim, na pesquisa quantitativa considera-se a possibilidade de quantificação em tudo, o que traduz em números as informações e as opiniões com a finalidade de classificar e analisar o objeto de estudo em questão, com a utilização de técnicas estatísticas.

\section{Desenvolvimento}

\subsection{Características dos Métodos Quantitativos e Qualitativos}

Ainda na onda das Ciências Naturais, a Química, a Física, a Biologia, entre as várias outras ciências derivadas, as Ciências Médicas misturam-se com o entendimento de métodos quantitativos ou explicativos, Turato (2005), ou seja, tem como característica marcante a clareza nas explicações dos fatos.

Para Silva (2010), o método quantitativo comumente é o observável, objetivo e possível de mensurar, já para Marujo (2013), pesquisa quantitativa demonstra-se como dedutiva, objetiva, que testa teorias e segue padrões positivistas.

Segundo Günther (2006) a pesquisa de natureza quantitativa tem características como:

- Controle máximo sobre o experimento, inclusive produzindo ambientes artificiais com o objetivo de reduzir ou eliminar a interferência de variáveis interferentes e irrelevantes;

- O pesquisador se relaciona com o objeto de estudo de forma neutra e objetiva, não levando em conta seus sentimentos e percepções;

- Crenças e valores pessoais não são compreendidos como fontes de influências no âmbito dos estudos científicos;

- Os dados coletados são avaliados por uma linguagem matemática, o qual faz o uso das análises estatísticas e das teorias de probabilidade para explicar os fenômenos.

De acordo com as referidas características, o método quantitativo tem a linguagem matemática como a base na interpretação dos dados coletados, e almeja por resultados que se relaciona com a realidade empírica e as teorias que dão suporte ao estudo.

Diante desta linguagem matemática Minayo \& Sanches (1993), afirma que o método quantitativo se adjetiva em ser capaz de descrever, representar ou interpretar a multidiversidade de seres vivos e suas inter-relações. Tendo atuação em níveis de realidade, onde os dados se apresentam aos sentidos observáveis. 
Silva (2010) alerta o pesquisador a ter cautela ao utilizar a linguagem matemática, pois ela carece estar adequada ao objeto de estudo, para que o fenômeno seja explicado corretamente.

Este mesmo autor, completa que o pesquisador precisa possuir características no manuseio do método quantitativo, o que o torna apto na percepção de quando o objeto se afasta, tornando-se neutro e controlador, no decorrer do estudo.

As características do âmbito da teoria qualitativa, Turato (2005), da sua contribuição, quando pontua que o pesquisador se firma na busca de significados dos fenômenos, fatos, eventos, sentimentos entre outros, pois exerce função organizadora dos seres humanos, o pesquisador utiliza-se de termos indutivos, subjetivos, interpretação e compreensão dos fenômenos. A metodologia qualitativa destaca as seguintes características:

- Procura nos significados das coisas descrições construídas no ambiente social e é definida como subjetiva;

- Possuem propriedades não consolidada estruturalmente, possuem riquezas em sua totalidade enfatizando as interações;

- No apanhado da coleta de dados qualitativos conseguem respostas de maneiras variadas semi ou não estruturadas;

- As ferramentas de estudos são indutivas, e observa a não generalização no alcance dos resultados.

\subsection{Vantagens e desvantagens na adoção das abordagens quantitativa e qualitativa}

Nas pesquisas cientificas, para a concretização do conhecimento com rigor cientifico são utilizadas abordagens metodológicas, dentre elas cabe destacar algumas vantagens e desvantagens que ocorre quando o pesquisador faz sua opção na escolha do método.

O que difere os paradigmas quantitativo e qualitativo, está muito distante do uso dos instrumentos e técnicas adotados pelo pesquisador, pois a escolha de um instrumento em detrimento de outro, pode levar a um entendimento equivocado por parte da escolha da abordagem feita pelo cientista, conforme explanado por Tanaka \& Melo (2001).

Para possibilitar a visualização das discrepâncias mediante as abordagens quantitativa e qualitativa, pontuando as vantagens e desvantagens entre os métodos, será apresentado abaixo um quadro explicativo, elaborado pelos autores Tanaka \& Melo (2001).

QUADRO 1: Vantagens e Desvantagens das Abordagens Metodológicas

\begin{tabular}{|c|c|c|}
\hline $\begin{array}{l}\text { ABORDAGEM } \\
\text { ASPECTOS }\end{array}$ & QUANTITATIVA & QUALITATIVA \\
\hline VANTAGENS & $\begin{array}{l}\text { • POSSIBILITA A ANÁLISE } \\
\text { DIRETA DOS DADOS } \\
\text { • TEM FORÇA } \\
\text { DEMONSTRATIVA } \\
\text { • PERMITE GENERALIZAÇÃO } \\
\text { PELA REPRESENTATIVIDADE } \\
\text { - PERMITE INFERÊNCIA PARA } \\
\text { OUTROS CONTEXTOS }\end{array}$ & $\begin{array}{l}\cdot \text { PERMITE INTERAÇÃO } \\
\text { • CONSIDERA A } \\
\text { SUBJETIVIDADE DOS SUJEITOS } \\
\text { • PERMITE COMPREENDER } \\
\text { RESULTADOS } \\
\text { INDIVIDUALIZADOS } \\
\text { • PERMITE COMPREENDER A } \\
\text { DINÂMICA INTERNA DE } \\
\text { PROGRAMAS E ATIVIDADES } \\
\text { • PERMITE COMPREENDER } \\
\text { MÚLTIPLOS ASPECTOS DOS }\end{array}$ \\
\hline
\end{tabular}




\begin{tabular}{|c|c|c|}
\hline & & $\begin{array}{l}\text { PROGRAMAS E/OU SERVIÇOS } \\
\text { • PERMITE AVALIAR } \\
\text { RESULTADOS DIFUSOS E NÃO- } \\
\text { ESPECÍFICOS }\end{array}$ \\
\hline DESVANTAGENS & $\begin{array}{l}\text { • SIGNIFICADO É SEMPRE } \\
\text { SACRIFICADO EM } \\
\text { DETRIMENTO DO RIGOR } \\
\text { MATEMÁTICO EXIGIDO PELA } \\
\text { ANÁLISE } \\
\text { • NÃO PERMITE ANÁLISE DAS } \\
\text { RELAÇÕES } \\
\text { • OS RESULTADOS PODEM SER } \\
\text { CONSIDERADOS COMO } \\
\text { VERDADE ABSOLUTA }\end{array}$ & $\begin{array}{l}\text { • PODE CONDUZIR A UMA } \\
\text { EXCESSIVA COLETA DE DADOS } \\
\text { • DEPENDE DE UMA } \\
\text { CAPACIDADE MAIOR DE } \\
\text { ANÁLIIE POR PARTE DO } \\
\text { AVALIADOR } \\
\text { • EXIGE MAIOR USO DO } \\
\text { RECURSO TEMPO }\end{array}$ \\
\hline
\end{tabular}

FONTE: Tanaka \& Melo ( 2001,p. )

\section{METODOLOGIA}

Abordagens sobre os métodos quantitativos e qualitativos, descritos nos artigos escolhidos de modo aleatório nas bases Capes, Google acadêmico, que consiste na busca de entendimento dos autores na escolha do método para a pesquisa.

Conforme citado anteriormente o foco deste estudo encontra se nos métodos quantitativos, mas devido ao entrelaçamento entre os dois métodos será também referenciado o método qualitativo.

No texto de Queiroz (2013), intitulado como Pesquisa quantitativa e pesquisa qualitativa: Perspectivas para o campo da etnomusicologia, 0 autor aponta a sinergia entre os métodos quantitativos e qualitativos. Tendo como objetivo refletir sobre as aplicações destas duas abordagens metodológicas nos estudos etnomusicológicos.

No entanto, Queiroz (2013), enfatizou a importância na consolidação total da pesquisa das abordagens quantitativa e qualitativa, que são vistas como complementares e levam a dimensões mais abrangentes para a criação do trabalho, tornando o capaz de compreender a complexidade dos estudos no campo das ciências humanas e até mesmo nas ciências naturais.

Vindo do campo das ciências naturais os estudos etnomusicológicos, como em outros campos das ciências humanas, a pesquisa quantitativa e a pesquisa qualitativa tende a construir juntas os contextos das investigações, uma vez que, suas abordagens são acima de tudo, complementares.

Para o etnomusicólogo este entrelace facilita a concretização e a apresentação do trabalho de maneira contextualizada com a realidade da pesquisa.

$O$ artigo de Gomes e Araujo ( $S / D)$, nomeado Pesquisa QuantiQualitativa em Administração: Uma Visão Holística do Objeto em Estudo, tem como objetivo evidenciar a relevância de metodologias quanti-qualitativas no campo da administração como uma forma de se obter um entendimento mais acurado dos objetos estudados.

No decorrer deste artigo os autores levantam um forte debate envolvendo as metodologias utilizadas nas pesquisas cientificas, sendo que as 
metodologias quantitativas tradicionais, alicerçada no pensamento positivista, o qual não alcança todas as expectativas dos cientistas, uma vez que as pessoas transitam em um mundo com alto grau de complexidade.

Por outra vertente, as metodologias qualitativas alternativas, firmadas na ideologia interpretativa, tem seu crescimento no cenário científico, principalmente no campo das ciências sociais, as quais trazem em seu entendimento a proposta de preencher as lacunas deixadas pela ortodoxia metodológica.

Desta forma, a propensão na ciência no que tange às metodologias de pesquisa, é a aplicação de abordagens variadas, Gomes e Araújo embasados em alguns autores deixa a seguinte contribuição, que tanto o paradigma positivista quanto o interpretativo não chegam ao sucesso isoladamente, necessitando de ferramentas apropriadas para as mais diferentes questões de pesquisa.

Os autores argumentam a construção de um método quanti-qualitativo que consiga agregar aspectos quantitativos e qualitativos, e de acordo com uma visão extrema e oposta. Eles afirmam que esta separação entre quantitativo e qualitativo é maligno para ambos os paradigmas, e especialmente para o avanço da ciência.

Günther (2006), deixa sua contribuição com estudo que leva o nome de Pesquisa Qualitativa Versus Quantitativa: Esta é a Questão? O autor assume uma postura ecumênica diante de estudiosos quantitativos e qualitativos, abordando os ambos paradigmas argumentando suas vantagens e desvantagens e também seus pontos positivos e pontos negativos.

Corroborando com os autores Ribeiro e Filho (2007), Günther também enfatiza que o método escolhido deve alinhar a pergunta que determina a pesquisa, explana sobre as diferenciações entre os métodos de pesquisa quantitativa e qualitativa, e em seguida enfatiza a complexidade da pesquisa qualitativa e discute também sobre critérios de qualidade da pesquisa quantitativa.

O autor critica a dicotomia de Dilthey ao afirmar "explicamos a natureza, compreendemos a vida mental" os seres humanos e, portanto, sua vida mental como parte da natureza, esta constante conexão simultânea com a natureza em um esforço de explicação e compreensão, que resultam uma da outra.

Para Günther os construtos explicação e compreensão, embutido na pesquisa cientifica derivam uma da outra, de forma enfática impossível o sucesso de uma sem a outra, vistas então por este autor como complementares.

No esquema de construção do conhecimento não seria aconselhável ao pesquisador fazer opção entre os métodos quantitativos e qualitativos, e sim utilizar as inúmeras abordagens inerentes a cada método, alinhando a questão de sua pesquisa a um mínimo de tempo que alcance os resultados favorável a compreensão do fenômeno para o crescimento do bem-estar social..

Ainda na linha de autores que defendem a conexão entre os métodos quantitativos e qualitativos os autores Minayo e Sanches (1993), que apesar da longa data citada de publicação ainda conseguem manter atualizadas as explanações abordadas no artigo de sua autoria que tem como título Quantitativo-Qualitativo: Oposição ou Complementaridade? Este artigo surgiu de discussões das atividades curriculares do Curso de Pós-Graduação em Saúde Pública da Escola Nacional de Saúde Pública (Ensp), Fundação Oswaldo Cruz (Fiocruz) — os denominados Seminários Avançados de Teses. 
Os autores tiveram o ensejo de mostrar as potencialidades e limitações das abordagens quantitativa e qualitativa que estão sendo utilizadas nos estudos apresentados, seus instrumentos servem para Saúde Pública, especialmente no que tange acercar da realidade observada.

De acordo com outros autores citados neste estudo, confirma-se que na ausência de uma das duas teorias, no sentido de suficiência na compreensão completa da realidade, afirmando que o método para ser considerado bom, terá que permitir a elaboração correta dos dados, ajudar a exprimir sobre a dinâmica da teoria.

Outrossim, é apropriar do objeto de estudo e de oferecer elementos teóricos que facilitem sua análise, o cuidado na escolha do método é fundamental, pois este tem que ser operacionalmente realizável.

Contudo, chegaram à conclusão de que ambas as teorias envolvendo os métodos quantitativos e qualitativos são indispensáveis, no entanto em diversas situações são ineficientes para alocar toda realidade observada.

Fortalecendo ainda que os dois métodos descritos são complementares de acordo com o planejamento da investigação, estão em conformidade, levando em consideração que o método é a engrenagem do conhecimento cientifico o qual busca e a articulação entre uma teoria e uma realidade empírica, que além de sua postura instrumental também é visto como a vida do conteúdo.

Marujo (2013), produziu o artigo com o título a Pesquisa em Turismo: Reflexões sobre as Abordagens Qualitativa e Quantitativa. Demonstram que o setor de turismo pode utilizar das duas abordagens metodológicas, pois trata-se de um fenômeno de múltiplas dimensões. As abordagens quantitativa e qualitativa podem usadas em distintas etapas do esquema de uma investigação em turismo.

No setor de turismo o entrelaçamento das abordagens é pertinente na construção da pesquisa científica, que possibilitam acurácia nas interpretações e exatidão do fenômeno. Os autores enfatizam que a escolha deve ser feita de acordo com os anseios específicos do seu projeto investigativo.

No setor de turismo a combinação dos dois métodos tende a colaborar em um maior refinamento e consequentemente eleva o nível de riqueza embutido na pesquisa cientifica. Assim os autores deste artigo também contemplam o entrelaçamento dos estudos que suportam a utilização das duas abordagens para responder a problemática abordada na pesquisa.

Os autores Leite, Viana e Pereira (2006), no artigo intitulado como Métodos Quantitativos na avaliação da Capes: Uma Pesquisa Bibliográfica, os pesquisadores busca identificar os métodos quantitativos já aplicados à avaliação da CAPES.

O método é baseado em pesquisa bibliográfica em sistemas de busca, currículos Lattes, revistas nacionais A e sistema Google Scholar. Os artigos alcançados foram agrupados segundo os métodos quantitativos utilizados e, em seguida comparados.

Neste estudo verificou a relevância de revistas especializadas e encontrar possíveis tendências da utilização de métodos quantitativos.

Possibilitou localizar um elevado conglomerado com foco na utilização destes métodos na área de ciências exatas, mostrando escassez de outras áreas do conhecimento.

Tal afirmação se fortalece na quantidade de artigos obtidos evidenciando áreas de proximidade com os estudiosos de métodos quantitativos. A comparação do método exposto nos artigos levantados, 
facilitou o agrupamento em três classes à saber, estatística descritiva, inferência estatística e métodos e multicritérios.

Este agrupamento enfatiza critérios que fortalece a opção pelo método quantitativo, com destaque para o aumento da utilização da classe dos multicritérios, permitindo o melhor entendimento da avaliação da Capes.

Os autores Ribeiro e Filho (2007), dão ênfase no método quantitativo, de posse da produção do artigo Aplicação dos Métodos Quantitativos em Auditoria: Propostas para Otimizar Procedimentos e Reduzir Riscos.

Tendo como objetivo investigar a importância de aplicar técnicas estatísticas em atividades de auditoria, em adição a outros recursos quantitativos, para que o auditor independente possa executar procedimentos com mais segurança e emitir o seu parecer com o mínimo de risco possível.

Segmento como a auditoria para realizações de suas atividades passa por restrições de tempo e recursos financeiros, o auditor não consegue conferir todos os registros e transações com estas limitações.

Então diante destes percalços faz se necessário o uso ferramentas que consistem na diminuição dos riscos de emitir informações inadequadas acerca das demonstrações contábeis, relativa à empresa avaliada, a qual busca estratégias com alto nível de confiabilidade no intuito de proteger seus ativos de fraudes ou qualquer tipo de erros na análise de seus resultados.

No entanto, os autores fazem a seguinte pergunta: Como minimizar este tipo de risco por meio de recursos estatísticos? Diante desta interrogativa viabiliza a escolha do método adequado ao seu objeto de estudo.

Que neste caso foi o método quantitativo que abarcando recursos simples, como a regressão linear, conseguiu demonstrar que o uso de ferramentas estatísticas realmente pode contribuir com o auditor a localizar indícios de erros e fraudes nas demonstrações contábeis

Outra contribuição vem a partir dos autores Fiates, Serra e Martins (2013), através do artigo nomeado A Aptidão dos Pesquisadores Brasileiros Pertencentes aos Programas de Pós-Graduação Stricto Sensu em Administração para Pesquisas Quantitativas, buscou analisar a competência de docentes de programas de pós-graduação em administração, recomendados pela Capes, no uso de métodos quantitativos diversos.

Os autores destacaram a complexidade do saber na área da Administração, apontando a distância na posse de seu caráter multidisciplinar, contudo possui a complexidade de seu objeto de estudo.

Diante deste cenário, pesquisadores que se interesse na produção de pesquisas que podem levar a geração de um novo conhecimento, corroborou com a evolução da área da administração. Desta forma, ocorre um forçamento para alcançar os métodos de pesquisa que sejam alinhados aos seus interesses.

Os métodos quantitativos transitam entre os métodos, e apresentam uma enorme variação de alternativas para o tratamento de dados, mesmo que seus resultados sejam vistos como confiáveis e generalizáveis, em situações que assegurem sua importância acadêmica, sua utilização dependerá das competências dos pesquisadores.

Os resultados deste estudo, confirma a importância em disponibilizar sistematicamente disciplinas ou cursos complementares da disciplina de métodos quantitativos não somente para alunos de programas da Administração, mas para todos os alunos que possuam interesse e reconhecimento da necessidade de sua utilização. 


\section{CONSIDERAÇÕES FINAIS}

De posse do dos artigos alcançados para produção desta pesquisa, os quais foram selecionados aleatoriamente nas bases já mencionadas, foi possível a compreensão sobre a abordagem metodológica quantitativa nas pesquisas cientificas.

Porém, é notório que os pesquisadores estão em crescente adoção da complementaridade das abordagens metodológicas, que apesar de terem características peculiares, ambas apresentam vantagens e desvantagens dentro do desenvolvimento do conhecimento cientifico.

No entanto, o método deve ser apresentado como um facilitador o qual busca atender as expectativas do pesquisador rumo ao alcance de questões que possibilitem compreender o objeto de investigação adotado na pesquisa cientifica, de acordo com os textos explanados para aplicabilidade dos métodos, fica evidente a tendência em utilizar os métodos quantitativos e qualitativos de maneira que eles possam se complementar e suprir possíveis deficiências que venham apresentar.

Os autores Queiroz (2013), Gomes e Araújo (S/D), Günther (2006), Minayo e Sanches (1993), Marujo (2013), corroboram na metodologia de pesquisa cientifica apostando na complementaridade entre as abordagens quantitativas e qualitativas, justificando com as vantagens e desvantagens entre as duas teorias e especialmente no que tange alcançar o objeto de estudo em curto tempo, apontam as características entre os métodos e na capacidade de suprir lacunas um do outro.

Leite, Viana e Pereira (2006), Fiates, Serra e Martins (2013), Ribeiro e Filho (2007), apontam justificativas que norteiam 0 uso dos métodos quantitativos, dialogando sobre sua objetividade, precisão e sua capacidade de multivariedade.

Assim conclui-se, que transitam entre os pesquisadores na produção de seus arranjos científicos, a complementaridade das duas abordagens quantitativa e qualitativa no intuito de suprir com exatidão a compreensão a realidade de forma holística no âmbito cientifico. 


\title{
APPROACHES Methodological IN THE FIELD OF SCIENTIFIC RESEARCH
}

\begin{abstract}
The growing demand for new knowledge lead in numerous movements in the field of scientific research, regarding the choice of methodology to be used in the understanding of the phenomena studied. In order to present an understanding of the quantitative approach in scientific research, results found that strengthen the intertwining of the two methodological approaches that guide the universe of scientific research. Yet neither method can be considered better at the expense of another, because the advantages and disadvantages in the use of the methods and also the latent features that differ from the methods and their characteristics are considered. These considerations is the relevance highlighted in this study, quantitative and qualitative approaches have been perceived as complementary among researchers in the scientific research universe.
\end{abstract}

Keywords:. Quantitative Methods, Complementarity, Scientific Research

\section{Referências}

BIGNARDI, F. A. (2009). Reflexões sobre a pesquisa qualitativa \& quantitativa: Maneiras complementares de apreender a realidade. Acesso em 05/04/2016. http://www.comitepaz.org.br/download/PESQUISA\%20QUALITATIVA.pdf

FAKIS, A. et al. Quantitative analysis of qualitative information from interviews: a systematic literature review. Journal of Mixed Methods $\begin{array}{llllll}\text { Research, } & \text { v. 8, } & \text { n. 2, } & \text { p. }\end{array}$ www.spell.org.br/documentos/ver/.../quantitative-methods- in...research.

GABRIEL, Marcelo Luiz. Métodos quantitativos em ciências sociais: sugestões para elaboração do relatório de pesquisa. Desenvolvimento em Questão, v. 12, n. 28, p. 348-369, 2014.https://www.revistas.unijui.edu.br/index.php/desenvolvimentoemquesta o/article/viewFile/2887/3430.

GÜNTHER, H. (2006). Pesquisa qualitativa versus pesquisa quantitativa: esta é a questão. Psicologia: teoria e pesquisa, 22(2), 201-210. http://www.scielo.br/pdf/ptp/v22n2/a10v22n2.pdf.

QUEIROZ, L. R. S. (2006). Pesquisa quantitativa e pesquisa qualitativa: Perspectivas para o campo da etnomusicologia. Claves, (2). files.wendelandrade.webnode.com.br/.../Pesquisa\%20qualitativa\%20versus $\% 20 q u a n t i t$ 
LEITE, M. F, B., Viana, A. B. N., \& Pereira, G. G. (2010). Métodos quantitativos na avaliação da CAPES: uma pesquisa bibliográfica. FACEF Pesquisa-Desenvolvimento e Gestão, 9(2). ojs.rbpg.capes.gov.br/index.php/rbpg/article/download/445/pdf

MACEDO RIBEIRO, J., \& Dias Filho, J. M. (2007). Aplicação de métodos quantitativos em auditoria: propostas para otimizar procedimentos e reduzir riscos. Revista de Contabilidade e Organizações, (1), 43. www.revistas.usp.br/rco/article/view/34696 ribeiro e filho

MARUJO, N. (2013). A pesquisa em Turismo: Reflexões sobre as Abordagens Qualitativa e Quantitativa. Revista de investigación en turismo y desarrollo local, 6(14). www.eumed.net/rev/turydes/14/pesquisaturismo.pdf marujo

MINAYO, M. C. S., \& Sanches, O. (1993). Quantitativo-qualitativo: oposição ou complementaridade. Cadernos de saúde pública, 9(3), 239-262.

unisc.br/portal/.../quantitavivo_qualitativo_oposicao_ou_complementariedad e .pdf

SAMPIERI, R. H.; COLLADO, C. F.; LUCIO, M. P. B. Metodologia de

pesquisa. 5. ed. Porto Alegre: Penso,

2013. www.grupoa.com.br/livros/pesquisa.../metodologiade- esquisa/9788565848282

SILVA, G. C. R. F. (2010). O método científico na Psicologia: Abordagem qualitativa e quantitativa. Psicologia.com.Pt. Jornal dos psicólogos. www.psicologia.pt/artigos/textos/A0539.pdf silva

TANAKA, O. Y., \& Melo, C. (2001). Avaliação de programas de saúde do adolescente: um modo de fazer.

Edusp.

www.scielo.br/scielo.php?script=sci_arttext\&pid=S0102-

311X2015000300654 tanaka

TURATO, E. R. (2005). Métodos qualitativos e quantitativos na área da saúde: definições, diferenças e seus objetos de pesquisa. Qualitative and quantitative methods in health: definitions, differences and research subjects. Rev
www.scielo.br/pdf/rsp/v39n3/24808.pdf

Saúde

Pública,

39(3), 507-14. 